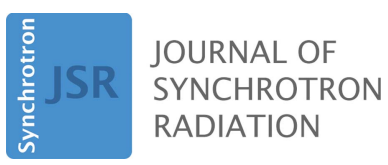

ISSN 1600-5775

Received 15 December 2020

Accepted 18 March 2021

Edited by A. Momose, Tohoku University, Japan

Keywords: 3D elemental distinguishing; dual-energy focal stacks imaging method; X-ray microscopy; STXM.

Supporting information: this article has supporting information at journals.iucr.org/s

\section{Three-dimensional fast elemental mapping by soft $X$-ray dual-energy focal stacks imaging}

\author{
Limei Ma, ${ }^{\mathrm{a}}$ Zijian Xu, ${ }^{\mathrm{a}}$ Zhi Guo, ${ }^{\mathrm{a}}$ Benjamin Watts, ${ }^{\mathrm{b}}$ Jinyou Lin, ${ }^{\mathrm{a} *}$ Xiangzhi Zhang ${ }^{\mathrm{a} *}$ \\ and Renzhong Tai $^{\mathrm{a} *}$

\begin{abstract}
aShanghai Synchrotron Radiation Facility, Shanghai Advanced Research Institute, Chinese Academy of Sciences, Shanghai 201204, People's Republic of China, and ${ }^{\mathbf{b}}$ Swiss Light Source, Paul Scherrer Institute, 5232 Villigen,
\end{abstract} \\ Switzerland. *Correspondence e-mail: linjinyou@zjlab.org.cn, zhangxiangzhi@zjlab.org.cn, tairenzhong@zjlab.org.cn
}

The three-dimensional (3D) dual-energy focal stacks (FS) imaging method has been developed to quickly obtain the spatial distribution of an element of interest in a sample; it is a combination of the 3D FS imaging method and twodimensional (2D) dual-energy contrast imaging based on scanning transmission soft X-ray microscopy (STXM). A simulation was firstly performed to verify the feasibility of the 3D elemental reconstruction method. Then, a sample of composite nanofibers, polystyrene doped with ferric acetylacetonate [Fe(acac)3], was further investigated to quickly reveal the spatial distribution of $\mathrm{Fe}(\mathrm{acac}) 3$ in the sample. Furthermore, the data acquisition time was less than that for STXM nanotomography under similar resolution conditions and did not require any complicated sample preparation. The novel approach of 3D dualenergy FS imaging, which allows fast 3D elemental mapping, is expected to provide invaluable information for biomedicine and materials science.

\section{Introduction}

Porous electrospun nanofibers, as superior one-dimensional nanostructures, have been applied in many fields such as electrochemical anodization, catalysis, absorption, etc. (González del Campo et al., 2018; Zhang, Han et al., 2010; Jo et al., 2018). Recent works on electrospun nanofibers have dealt with the incorporation of different types of inorganic nanoparticles into this material for diverse functions (Lin, Shang et al., 2012; Miyamoto et al., 2017). Iron acetylacetonate [Fe(acac)3, acetylacetonate(acac)] dispersed into polymer nanofibers make it attractive in magnetic devices and biomagnetic regimes (Mahapatra et al., 2013; Moatmed et al., 2019). Therefore, investigating composite nanofibers decorated with $\mathrm{Fe}(\mathrm{acac}) 3$ provides potential value in biomaterials and nanoscience.

Traditionally, nanoscale investigation of the internal structure within nanofibers has been possible using various techniques including soft X-ray and electron microscopies, which are limited to investigations of thin samples (Shenhar \& Rotello, 2003; Lin, Ding et al., 2012). For example, in transmission electron microscopy, sample preparation and mechanical slicing procedures are essential for thick samples, and scanning electron microscopy only enables surface or cross-section microstructures to be observed (McIntosh et al., 2005; Wei et al., 2017). Typically, nanotomography is the commonly used technique for the three-dimensional (3D) imaging-based scanning transmission soft X-ray microscope (STXM), which is still a challenge for thick, large size samples due to high absorption for rotation at large angles (Johansson et al., 2006; Schmid et al., 2014). In laminography, the tilted 
geometry becomes more difficult with higher-resolution (shorter focal length) Fresnel zone plates (FZPs), with a risk of collision with upstream optical elements (Witte et al., 2020). Focused ion beam milling combined with scanning electron microscopy (FIB-SEM) is also a powerful tool for observation of polymer 3D structures (Denk \& Horstmann, 2004). However, all of these methods are able to achieve elemental mapping when they are further combined with spectral microscopy.

The dual-energy contrast imaging method, developed on an STXM, is performed to achieve 2D elemental mapping (Ade et al., 1992; Jiang et al., 2010; Smit et al., 2008). In this method, the absorption of a certain element in the sample will abruptly increase when the incident X-ray energy changes from its pre-edge $E_{1}$ to the absorption edge $E_{2}$, while the absorption changes of other elements are so small that they can be neglected (Warp \& Dobbins, 2003; Zhang, Xu et al., 2010). Hitchcock et al. investigated 3D chemical mappings of a polyacrylate polyelectrolyte ionomer embedded with polystyrene microspheres at multiple photon energies (Hitchcock et al., 2008; Johansson et al., 2007). Yao et al. achieved the distributions of intracellular nanomaterials in three dimensions in macrophages by combining the dual-energy contrast imaging method and equally sloped tomography (Yao et al., 2018). This combined approach, however, needs image alignment and refocusing during each tomography rotation and therefore is more time-consuming for data acquisition at multiple energies.

Focal stacks (FS) imaging is an emerging three-dimensional reconstruction method extended towards STXM, making possible for of the order of hundreds of nanometres reconstruction of test samples in about an hour, thus providing a flexible and fast solution for 3D imaging (Streibl, 1985; Jochum, 1988). A series of 2D microscopic images are collected by moving a sample along the optical axis with a fixed step without rotation. After processing the nanoscale $2 \mathrm{D}$ image sequences with the improved variancebased FS algorithm we proposed, a 3D structured image can be quickly reconstructed (Ma et al., 2019). In previous work, we reconstructed the $3 \mathrm{D}$ image of a $4 \mu \mathrm{m}$-thick cellulose film embedded with $\mathrm{Fe}_{3} \mathrm{O}_{4}$ nanoparticles within about 66 min (Ma et al., 2019).

In this communication, we report the 3D dual-energy FS imaging method, which exploits the combination of FS imaging and the dual-energy contrast method to quickly generate 3D elemental mapping on the nanoscale. Firstly, a simulation experiment was

Figure 1 conducted to validate the feasibility of the method. It was further carried out to achieve the element spatial mapping in composite nanofibers. For the first time, the 3D dual-energy FS imaging method can be used to reconstruct porous polystyrene (PS) doped with $\mathrm{Fe}(\mathrm{acac}) 3$.

\section{Experimental setup and method}

The experimental setup of STXM, shown in Fig. 1(a), is used for dual-energy FS imaging, which enables microscopic images based on density, element and different contrast of samples to be acquired. A schematic of the 3D dual-energy FS imaging method is shown in Fig. 1(b). Before discussions on the 3D reconstruction, we analyze a line scan taken along an edge of the sample in the STXM image to obtain the near-edge X-ray absorption fine-structure spectroscopy (NEXAFS) spectra of the element, as well as the energies $E_{1}$ and $E_{2}$ (Ohmer et al., 2015; Nilsson et al., 2005). Two series of 2D image sequences are collected at $E_{1}$ and $E_{2}$, respectively. Next, we exploit the 3D FS algorithm to reconstruct 3D structure images under the two energy edges (Ma et al., 2019). The dual-energy ratiocontrast algorithm or $K$-edge subtraction algorithm is finally performed to elucidate detailed spatial distributions of the element of interest in the sample, so 3D mappings of the element can be seen clearly.
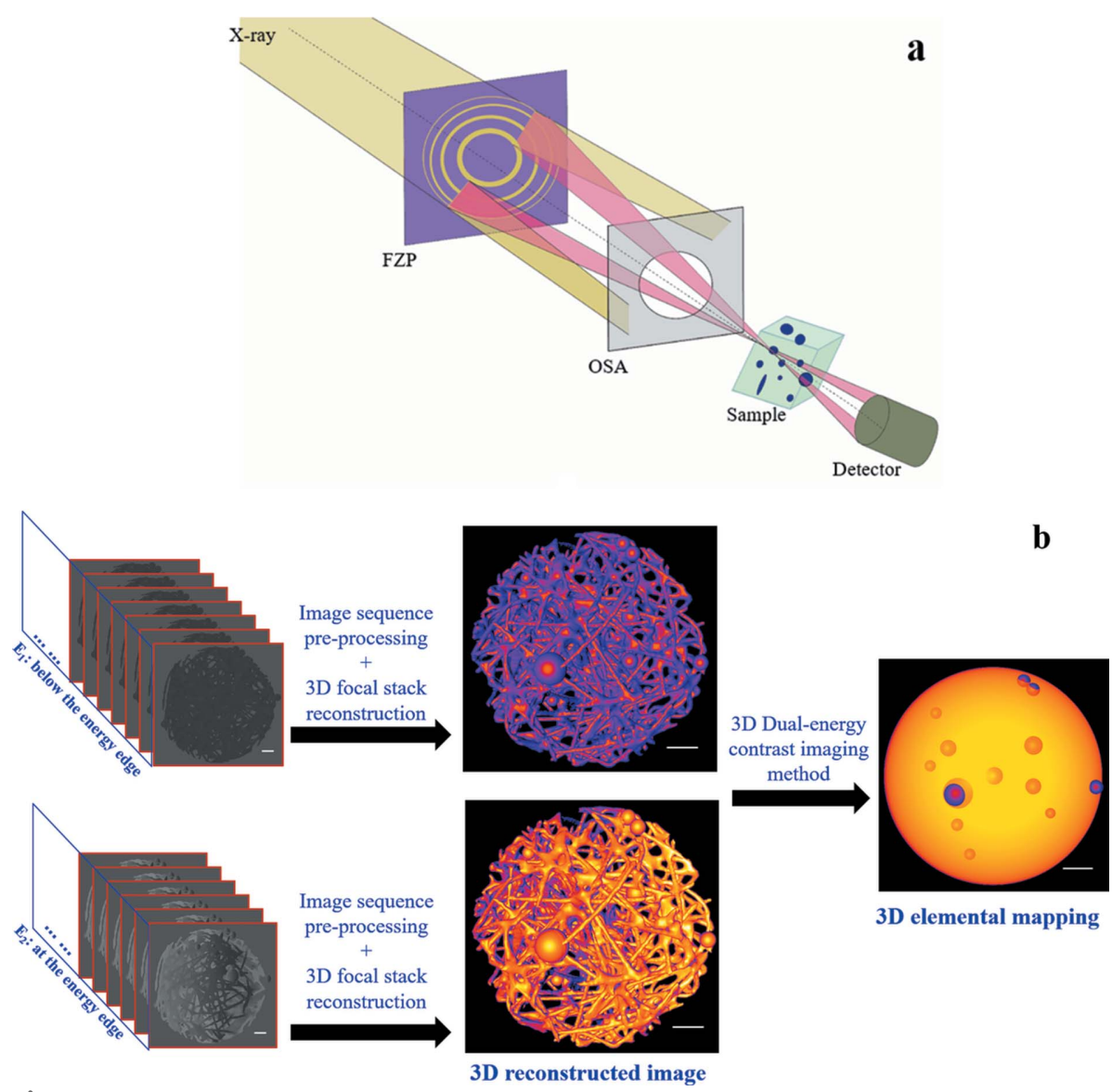

(a) Experiment setup for scanning transmission soft X-ray microscopy. (b) Schematic layout of the 3D dual-energy focal stacks (FS) reconstruction method. Scale bar: $500 \mathrm{~nm}$. 


\section{Simulation}

We conducted a dual-energy FS simulation of a modeled sample - a carbon cube randomly embedded with iron $(\mathrm{Fe})$ and manganese $(\mathrm{Mn})$ nanoparticles, ranging from 100 to $600 \mathrm{~nm}$ [Fig. 2(a)]. In the simulation, incident photon energies were $708 \mathrm{eV}$ and $700 \mathrm{eV}$ at the $\mathrm{Fe} L_{3}$ energy edge and preedge, respectively. The FZP has a diameter of $60 \mu \mathrm{m}$, a centralstop-radius of $24 \mu \mathrm{m}$ and an outermost zone width $\left(\Delta r_{n}\right)$ of $10 \mathrm{~nm}$, so the depth of focus (DOF) was calculated to be about $230 \mathrm{~nm}$. Fresnel-Kirchhoff diffraction and X-ray multi-slice propagation theories were used for acquisition of the STXM images (Goodman, 2005; Attwood, 2017; Maiden et al., 2012; Ma et al., 2019). Figs. 2(b)-2(c) show two examples of images of size $3 \mu \mathrm{m} \times 3 \mu \mathrm{m}$ collected at $E=708 \mathrm{eV}$ and $700 \mathrm{eV}$. Note that the signal from Fe particles at $700 \mathrm{eV}$ has been ignored since there is too much absorption change to be detected as can be seen in Fig. 2(c).

Then, the sample was moved along the optical axis with a step of $50 \mathrm{~nm}$ to sequentially collect 61 images at $708 \mathrm{eV}$ and $700 \mathrm{eV}$. Finally, 3D reconstructions at the two energy edges were computed by the improved FS algorithm. Fig. 2(d) shows a 3D image reconstructed at $708 \mathrm{eV}$, in which $\mathrm{Fe}$ and $\mathrm{Mn}$ particles are clearly seen; however, they are indistinguishable in the sample. Similarly, a 3D map at $700 \mathrm{eV}$ with FS analysis is shown in Fig. 2(e). Fig. 2(f) presents two different renderings in a combined map of the reconstructed image from $700 \mathrm{eV}$ and Fe nanoparticles from the dual-energy FS image, in which the distributions of the reconstructed structures are in agreement with that in the modeled sample. Due to the effect of color superposition, there is a slight color difference between $\mathrm{Mn}$ and the corresponding gray colormap. The method was further introduced to composite nanofibers to explore its 3D characterization applications in real samples.

\section{Experiment}

An imaging experiment on composite nanofibers was carried out at the PolLux beamline station, Swiss Light Source (Raabe et al., 2008). The porous-structured $\mathrm{Fe}(\mathrm{acac}) 3$ PS nanofibers [PS@Fe(acac)3] were produced by soaking pure PS nanofibers in a $10 \mathrm{wt} \% \mathrm{Fe}(\mathrm{acac}) 3$ ethanol solution overnight. Experimentally, the 2D dual-energy contrast imaging method was firstly performed to explore 2D distributions of $\mathrm{Fe}(\mathrm{acac}) 3$ in the composite fibers. Two STXM images acquired at the $705 \mathrm{eV}$ and $712 \mathrm{eV}$ energy edges are shown in Figs. 3(a) and $3(b)$, respectively (NEXAFS spectra are shown in Fig. S1 of the supporting information). By analysis using the dual-energy ratio-contrast algorithm, the result shows $2 \mathrm{D}$ distributions and accumulation of $\mathrm{Fe}(\mathrm{acac}) 3$, Fig. 3(c). According to the colorbar on the right, red represents high density of the sample, blue corresponds to low density, and dark blue means zero density. Close examination of the dual-energy contrast image, as indicated by the yellow arrows, shows there are cases where there appears to be accumulations of $\mathrm{Fe}(\mathrm{acac}) 3$ parti-
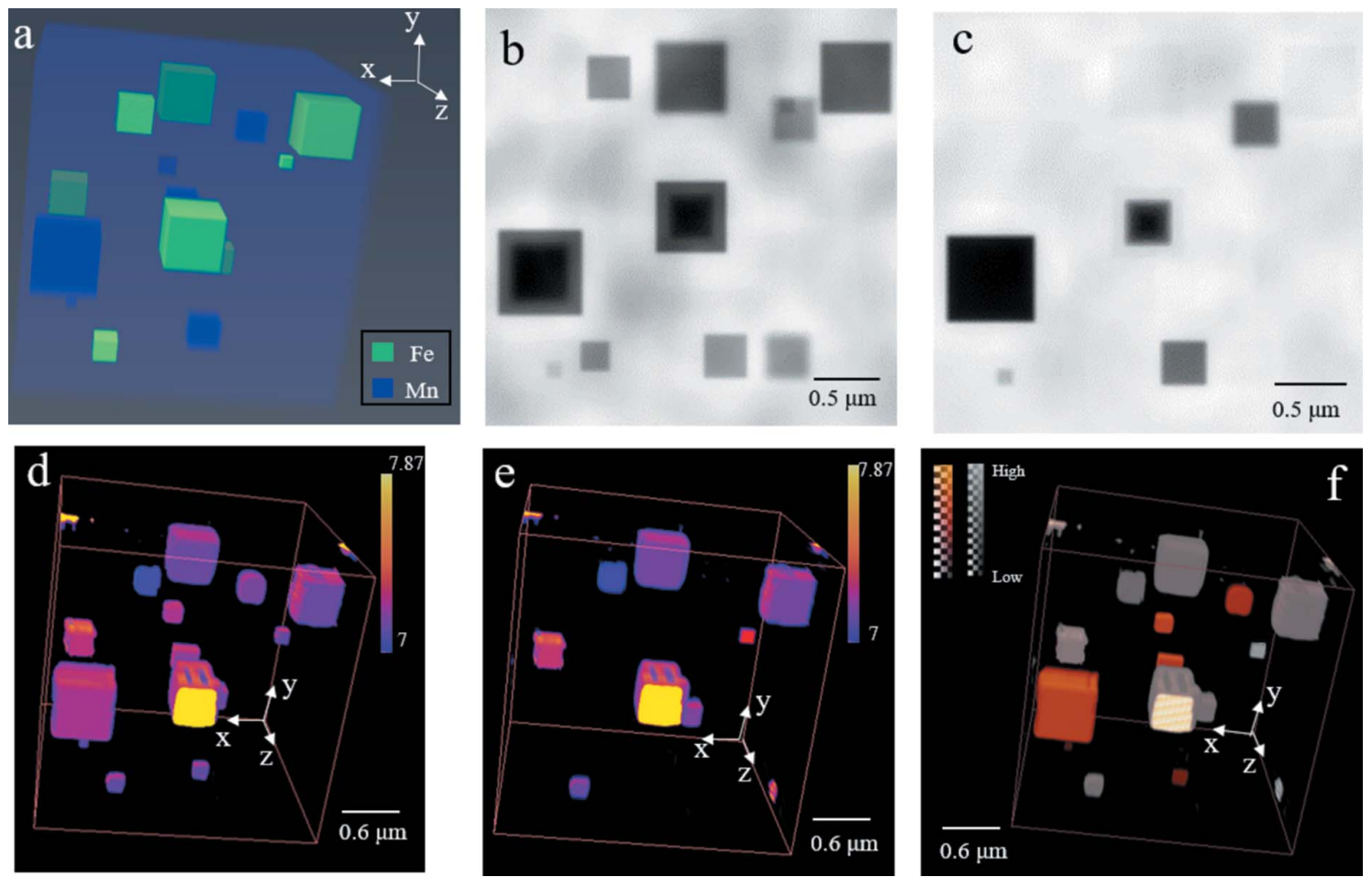

Figure 2

Simulation study with a modeled sample using the 3D dual-energy focal stacks imaging method. (a) The sample model in the simulation. The iron (Fe) and manganese $(\mathrm{Mn})$ cubic particles are randomly distributed inside the carbon matrix sample with sizes of 100 to $600 \mathrm{~nm}$. The green color represents Fe particles and blue cubes represent Mn. $(b, c) 2 \mathrm{D}$ STXM images acquired at $E=708 \mathrm{eV}$ and $E=700 \mathrm{eV}$, respectively. $(d, e) 3 \mathrm{D}$ reconstructed images of the sample at $708 \mathrm{eV}$ and $700 \mathrm{eV}$. The colorbar to the right represents the sample density, which is proportional to the optical density. Unit of colorbar: $\mathrm{g} \mathrm{cm}^{-3}$. ( $f$ ) 3D combined representations of Fe (gray color) and Mn (orange color) distributions. 

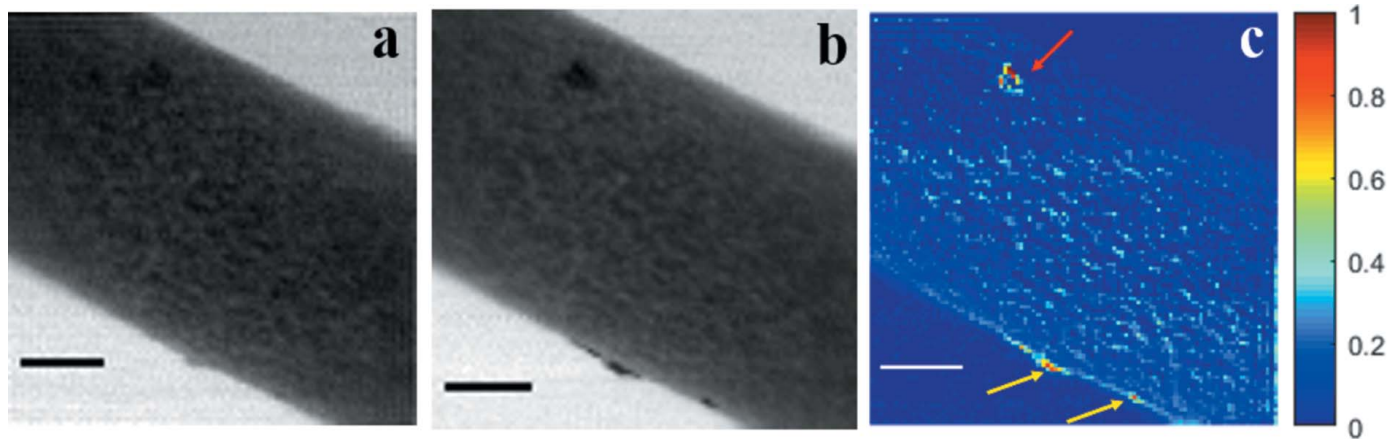

Figure 3

Soft X-ray STXM images and the dual-energy contrast images for the PS@Fe(acac) 3 composite nanofibers. $(a, b)$ STXM images collected at $E_{1}=705$ eV and $E_{2}=712 \mathrm{eV}$, respectively. (c) 2D dual-energy contrast image from analysis of $(a)$ and $(b)$. The scale bar is $1 \mu \mathrm{m}$.

cles on the sides of fibers. However, it is insufficient, as the red arrow indicates, to distinguish the positions of $\mathrm{Fe}(\mathrm{acac}) 3$ from the $2 \mathrm{D}$ structured image.

To quickly distinguish the spatial distribution of $\mathrm{Fe}(\mathrm{acac}) 3$ in the porous composite nanofibers, a 3D dual-energy FS experiment was carried out based on STXM for Fe(acac)3 spatial localization. In the experiment, $\Delta r_{n}$ of the FZP was $15 \mathrm{~nm}$ so the DOF was about $500 \mathrm{~nm}$. Taking into account the DOF and oversampling, the step size of the motor along the optical axis was set as $300 \mathrm{~nm}$. By moving the sample with a fixed step, 40 images were collected sequentially over $12 \mu \mathrm{m}$ along the optical axis to ensure each part of the structure was within the DOF in one of the $2 \mathrm{D}$ micrographs at $712 \mathrm{eV}$, as well as at $705 \mathrm{eV}$. The automatic image acquisition for FS experimental study was about $3 \mathrm{~h}$ without any extra operation or particular sample preparation, which is evidently faster than STXM-based nanotomography under the similar resolution condition. The 3D FS reconstructions were computed using the improved variance-based FS algorithm after image pre-processing of normalization, denoising, and image sequences alignment (Ma et al., 2019). The result shown in Fig. 4(a) is a 3D map of PS@Fe(acac)3 composite nanofibers reconstructed at $712 \mathrm{eV}$, the angle observation of which is consistent with that in Fig. 3. Colors in the image indicate the densities of fibers as shown by the fire scale colorbar in the upper right corner. Yellow means high density and blue corresponds to low density of the composite nanofiber. The 3D visualization video can be found in Movie 1 of the supporting information. In Movie 1, 3D structures of composite nanofibers are shown more clearly; however, it is impossible to differentiate the positions of $\mathrm{Fe}(\mathrm{acac}) 3$ in the porous fibers. It is hence desirable to combine with the dualenergy contrast imaging method.

By processing and analyzing the two 3D reconstruction images with the dual-energy ratio-contrast algorithm, spatial distributions of $\mathrm{Fe}(\mathrm{acac}) 3$ were obtained. Fig. 4(b) shows 3D mappings of $\mathrm{Fe}(\mathrm{acac}) 3$ from the same view as Fig. 4(a), in which the $\mathrm{Fe}(\mathrm{acac}) 3$ can be seen clearly (see Movie 2 of the supporting information). There are some accumulation and agglomerations, consistent with the SEM result in Fig. S2. Movie 2 clearly shows that the $\mathrm{Fe}(\mathrm{acac}) 3$ particles or large agglomerations are mostly distributed around surfaces or
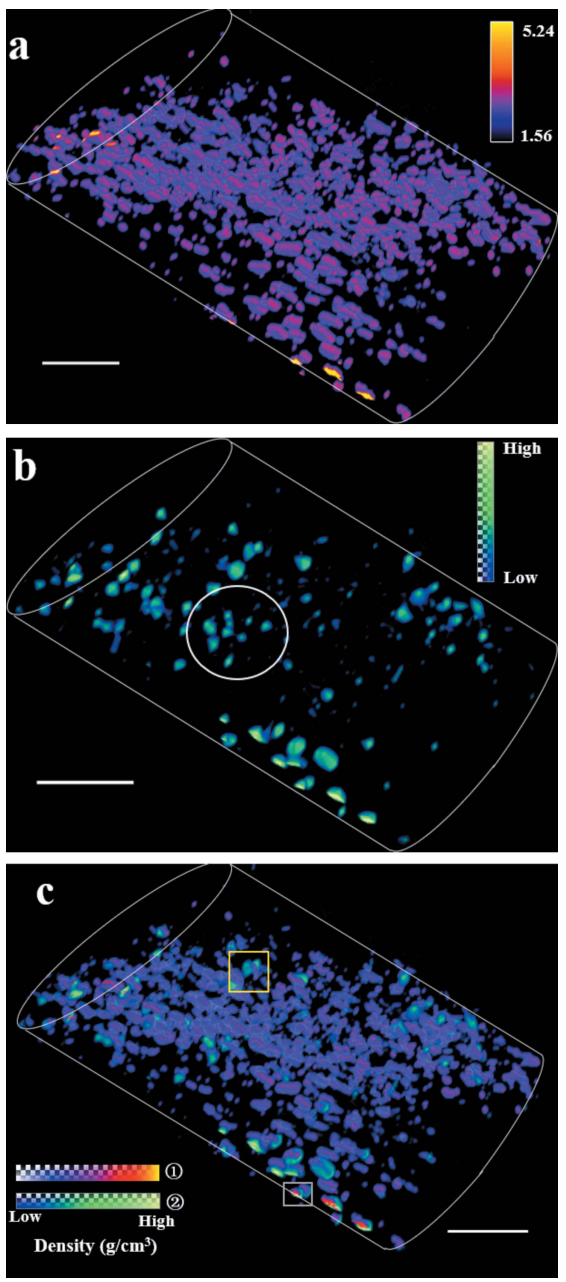

Figure 4

3D reconstruction of PS@Fe(acac)3 composite nanofibers and the spatial distribution of $\mathrm{Fe}(\mathrm{acac}) 3$. (a) 3D reconstruction of PS@Fe(acac)3 composite nanofibers. The colorbar to the right indicates the sample density. The larger the value, the higher the density value, and it is proportional to the optical density. Color scale is in $\mathrm{g} \mathrm{cm}^{-3}$. (b) Spatial distributions of $\mathrm{Fe}(\mathrm{acac}) 3$ after dual-energy FS reconstruction from the same angle observation of $(a)$. (c) Volume rendering of the nanofibers and distributions of $\mathrm{Fe}(\mathrm{acac}) 3$ simultaneously. The 'Temperature' colormap represents the fibers reconstructed from $E=705 \mathrm{eV}$ [legend (1)] and the 'VolrenGreen' colormap shows the Fe(acac)3 mapping [legend (2)]. Panel $(c)$ is in the same rotation view as $(a)$ and $(b)$. Gray and yellow boxes are two obvious examples showing Fe(acac) 3 inside the fibers. Scale bar: $1 \mu \mathrm{m}$. 
edges of the fibers, the accumulation of which is relatively less than that on the surface. For example, the $\mathrm{Fe}(\mathrm{acac}) 3$ particles marked by a white dotted circle in Fig. 4(b) are actually inside the nanofibers shown in Movie 2. Furthermore, we observed nanostructures of fibers and $\mathrm{Fe}(\mathrm{acac}) 3$ distributions simultaneously with a two-colormap combination [Fig. 4(c)]. The presentation was made by combining the $3 \mathrm{D}$ reconstruction image at $705 \mathrm{eV}$ and spatial $\mathrm{Fe}(\mathrm{acac}) 3$ mapping. It is clear that the distributions of $\mathrm{Fe}(\mathrm{acac}) 3$ in the nanofibers are varied. Taking two details as examples, nanoparticles in the gray box in Fig. 4(c) show the $\mathrm{Fe}(\mathrm{acac}) 3$ inside the fiber. Similarly, the yellow marked parts are also inside the fibers. In addition, the dose used to acquire this image sequence at $712 \mathrm{eV}$ was only $\sim 7.2 \times 10^{5}$ Gy (supporting information), which is typically lower than the corresponding full-field imaging or nanotomography (typical $10^{6}$ to $10^{8} \mathrm{~Gy}$ ) (Gros et al., 2005; Meyer-Ilse et al., 2001). Thus, radiation damage is a considerable advantage by STXM compared with full-field microscopy.

\section{Conclusion}

In conclusion, we have developed the three-dimensional dualenergy FS method for fast elemental mappings in a sample. The spatial elemental distribution is directly reconstructed from two series of image sequences using the improved variance-based focal stack algorithm and dual-energy ratiocontrast algorithm. Both simulation and applicable experimental results have indicated the feasibility of the 3D imaging method. Reconstructed $\mathrm{Fe}(\mathrm{acac}) 3$ particles were randomly distributed inside the porous composite nanofibers or on the surface. Compared with conventional nanotomography, the 3D dual-energy FS imaging method reduces by more than two-thirds of the time to achieve a similar resolution result. Thus, the advantages of the 3D dual-energy FS method are its quick, efficient and quantitative elementally reconstruction at nanoscale resolution, as well as no extra and particular sample preparation or labeling. It is expected that the $3 \mathrm{D}$ dual-energy FS method will offer broader characterization applications in nanomedicine, biological and other fields.

\section{Acknowledgements}

The authors thank PolLux beamline of Swiss Light Source and BL08U1A beamline of Shanghai Synchrotron Radiation Facility for providing beam time. They also thank Katharina Witte (Swiss Light Source, Paul Scherrer Institute) for assisting with the data acquisition.

\section{Funding information}

This work was supported by the National Natural Science Foundation of China (Nos. 51773221, 11875316 and U1832146) and the Youth Innovation Promotion Association CAS (Nos. 2017308 and 2019291).

\section{References}

Ade, H., Zhang, H., Cameron, S., Costello, C., Kirz, J. \& Williams, S. (1992). Science, 258, 972-975.
Attwood, D. (2017). Soft X-rays and Extreme Ultraviolet Radiation: Principles and Applications. Cambridge University Press.

Denk, W. \& Horstmann, H. (2004). PLoS Biol. 2, e329.

González del Campo, M. M., Darder, M., Aranda, P., Akkari, M., Huttel, Y., Mayoral, A., Bettini, J. \& Ruiz-Hitzky, E. (2018). Adv. Funct. Mater. 28, 1703048.

Goodman, J. W. (2005). Introduction to Fourier Optics. McGraw-Hill. Hitchcock, A. P., Johansson, G. A., Mitchell, G. E., Keefe, M. H. \& Tyliszcak, T. (2008). Appl. Phys. A, 92, 447-452.

Jiang, H., Song, C., Chen, C. C., Xu, R., Raines, K. S., Fahimian, B. P., Lu, C. H., Lee, T. K., Nakashima, A., Urano, J., Ishikawa, T., Tamanoi, F. \& Miao, J. (2010). Proc. Natl Acad. Sci. USA, 107, 11234-11239.

Jo, M. S., Park, G. D., Kang, Y. C. \& Cho, J. S. (2018). Nanoscale, 10, 13539-13547.

Jochum, L. (1988). X-ray Microscopy II, Vol. 56 of Springer Series in Optical Science, pp. 292-295 Springer.

Johansson, G. A., Dynes, J. J., Hitchcock, A. P., Tyliszczak, T., Swerhone, G. D. \& Lawrence, J. R. (2006). Microsc. Microanal. 12, 1412-1413.

Johansson, G. A., Tyliszczak, T., Mitchell, G. E., Keefe, M. H. \& Hitchcock, A. P. (2007). J. Synchrotron Rad. 14, 395-402.

Le Gros, M. A., McDermott, G. \& Larabell, C. A. (2005). Curr. Opin. Struct. Biol. 15, 593-600.

Lin, J., Ding, B., Yang, J., Yu, J. \& Sun, G. (2012). Nanoscale, 4, 176 182.

Lin, J., Shang, Y., Ding, B., Yang, J., Yu, J. \& Al-Deyab, S. S. (2012). Mar. Pollut. Bull. 64, 347-352.

Ma, L. M., Zhang, X. Z., Xu, Z. J., Späth, A., Xing, Z. J., Sun, T. X. \& Tai, R. Z. (2019). Opt. Express, 27, 7787-7802.

Mahapatra, A., Mishra, B. G. \& Hota, G. (2013). J. Hazard. Mater. 258-259, 116-123.

Maiden, A. M., Humphry, M. J. \& Rodenburg, J. M. (2012). J. Opt. Soc. Am. A, 29, 1606-1614.

McIntosh, R., Nicastro, D. \& Mastronarde, D. (2005). Trends Cell Biol. 15, 43-51.

Meyer-Ilse, W., Hamamoto, D., Nair, A., Lelièvre, S. A., Denbeaux, G., Johnson, L., Pearson, A. L., Yager, D., Legros, M. A. \& Larabell, C. A. (2001). J. Microsc. 201, 395-403.

Miyamoto, A., Lee, S., Cooray, N. F., Lee, S., Mori, M., Matsuhisa, N., Jin, H., Yoda, L., Yokota, T., Itoh, A., Sekino, M., Kawasaki, H., Ebihara, T., Amagai, M. \& Someya, T. (2017). Nat. Nanotechnol. 12, 907-913.

Moatmed, S. M., Khedr, M. H., El-dek, S. I., Kim, H.-Y. \& El-Deen, A. G. (2019). J. Environ. Chem. Eng. 7, 103508.

Nilsson, H. J., Tyliszczak, T., Wilson, R. E., Werme, L. \& Shuh, D. K. (2005). Anal. Bioanal. Chem. 383, 41-47.

Ohmer, N., Fenk, B., Samuelis, D., Chen, C. C., Maier, J., Weigand, M., Goering, E. \& Schütz, G. (2015). Nat. Commun. 6, 6045 .

Raabe, J., Tzvetkov, G., Flechsig, U., Böge, M., Jaggi, A., Sarafimov, B., Vernooij, M. G. C., Huthwelker, T., Ade, H., Kilcoyne, D., Tyliszczak, T., Fink, R. H. \& Quitmann, C. (2008). Rev. Sci. Instrum. 79, 113704.

Schmid, G., Zeitvogel, F., Hao, L., Ingino, P., Kuerner, W., Dynes, J. J., Karunakaran, C., Wang, J., Lu, Y., Ayers, T., Schietinger, C., Hitchcock, A. P. \& Obst, M. (2014). Microsc. Microanal. 20, 531536.

Shenhar, R. \& Rotello, V. M. (2003). Acc. Chem. Res. 36, 549561.

Smit, E. de, Swart, I., Creemer, J. F., Hoveling, G. H., Gilles, M. K., Tyliszczak, T., Kooyman, P. J., Zandbergen, H. W., Morin, C., Weckhuysen, B. M. \& de Groot, F. M. F. (2008). Nature, 456, 222225.

Streibl, N. (1985). J. Opt. Soc. Am. A, 2, 121-127.

Warp, R. J. \& Dobbins, J. T. (2003). Med. Phys. 30, 190-198.

Wei, M., Wan, J., Hu, Z., Peng, Z., Wang, B. \& Wang, H. (2017). Appl. Surf. Sci. 391, 267-274. 
Witte, K., Späth, A., Finizio, S., Donnelly, C., Watts, B., Sarafimov, B., Odstrcil, M., Guizar-Sicairos, M., Holler, M., Fink, R. H. \& Raabe, J. (2020). Nano Lett. 20, 1305-1314.

Yao, S., Fan, J., Chen, Z., Zong, Y., Zhang, J., Sun, Z., Zhang, L., Tai, R., Liu, Z., Chen, C. \& Jiang, H. (2018). IUCrJ, 5, 141-149.
Zhang, H., Han, J. S. \& Yang, B. (2010). Adv. Funct. Mater. 20, 15331550.

Zhang, X. Z., Xu, Z. J., Tai, R. Z., Zhen, X. J., Wang, Y., Guo, Z., Yan, R., Chang, R., Wang, B., Li, M., Zhao, J. \& Gao, F. (2010). J. Synchrotron Rad. 17, 804-809. 